\title{
Evaluation of Hepatocellular Carcinoma by Gadolinium- Based Contrast Media on MRI
}

\author{
Saman Sanaullah ${ }^{1 *} \quad$ Nazeeha Waseem $^{1} \quad$ Waqas Aleem $^{2} \quad$ Rehan Afsar $^{2} \quad$ Abid Ali $^{3}$ \\ 1. Medical Imaging Doctor, Department of Radiological Sciences and Medical Imaging, The University of \\ Lahore, Gujrat \\ 2.Lecturer, Department of Radiological Sciences and Medical Imaging, The University of Lahore, Gujrat \\ 3.Associate Professor, Faculty of Allied Health Sciences, The University of Lahore, Gujrat
}

\begin{abstract}
Hepatocellular carcinoma (HCC) is a big cause of death associated with cancer in the world and is the most common liver malignancy. More prevalence of chronic hepatitis virus (primarily chronic hepatitis B and hepatitis C) is attributed to high prevalence in Asia. Purpose: The purpose of this research was to test gadolinium-based contrast media for hepatocellular carcinoma on MRI.A total of 96 patients from Lahore who visited at radiology department of Al Razi Health Care Hospital from August 2020 to November 2020 for the evaluation of hepatocellular carcinoma by gadolinium based contrast media on MRI. Results: The study was conducted on 96 patients for the evaluation of hepatocellular carcinoma, out of which 62 were diagnosed with hepatocellular carcinoma. HCC have become a major burden in the world increasing mortality and morbidity. Early identification leads to early treatment and improved survival.
\end{abstract}

Keywords: HCC, HCV, USG, MRI, Gadolinium

DOI: $10.7176 / \mathrm{JHMN} / 84-05$

Publication date: December $31^{\text {st }} 2020$

\section{INTRODUCTION}

The leading cancer-related cause of death in the world is Hepatocellular Carcinoma (HCC) and the most common liver malignant. There have been globally a million deaths/year. A high predomen in Asia is associated with the highest prevalence of chronic hepatitis virus (mainly chronic hepatite B and hepatitis C).Consumption of alcohol is also a major risk factor in developing HCC. The essential precipitating factor of HCC is Metabolic syndrome and Nonalcoholic Steatohepatite (NASH).Unfortunately, HCC carries one of the highest mortality rates of solid organs cancers, with 5-years survival of less than $15 \%$. The main reason for poor prognosis is that it is usually diagnosed at advanced stage.

Most cases of HCC occur in people who already have signs and symptoms of chronic liver disease. Patients are presented with abdominal swelling due to ascites, yellowish skin, and right upper quadrant abdominal pain, and nausea and weight loss. (Michael Reid, 2017) The evaluation of both asymptomatic and symptomatic patients involved both blood testing and imaging evaluation. In past decades Biopsy was required for the conformation of HCC but now imaging evaluation especially MRI is irrefutably is enough for diagnosis of HCC. Imaging MRI is preferred over CT and Ultrasound because it has higher sensitivity and specificity among other modalities therefore it can highly differentiate HCC with regenerative nodules. Grossly HCC appears nodular tumor, nodules can be single or multiple and well circumscribed but non capsular. (Julius balogh, 2016)

\section{MODALITIES}

Ultrasound uses high energy waves to make pictures of internal organs and other tissues. Relatively stable and noninvasive modality of imagery is ultrasound. Doppler ultrasound is a special ultrasound procedure that helps the doctor to see and assess blood supply through the abdomen's arteries and veins and multiple organ systems. Ultrasound waves are interference with air or steam, and hence ultrasound is not the perfect imagery tool for the air-filled intestine or bowel organ. (Hina Arif-Tiwari, 2014), (Runyon, 2016) On ultrasound the HCC lesion shows the increased vascularity as branching pattern. Through Doppler thrombus can be differentiated from tumor in the portal vein. Power Doppler is three to five times more sensitive than color Doppler in demonstrating the invasion of tumor vascularity. If HCC is smaller than $3 \mathrm{~cm}$, the lesion appear as hypo echoic in comparative to neighboring tissue while larger lesion appear as hyper echoic with patchwork pattern with hypo echoic fibrous capsule. Any variation in image can be due to presence of fat, calcium and necrosis. (Hina Arif-Tiwari, 2014) 


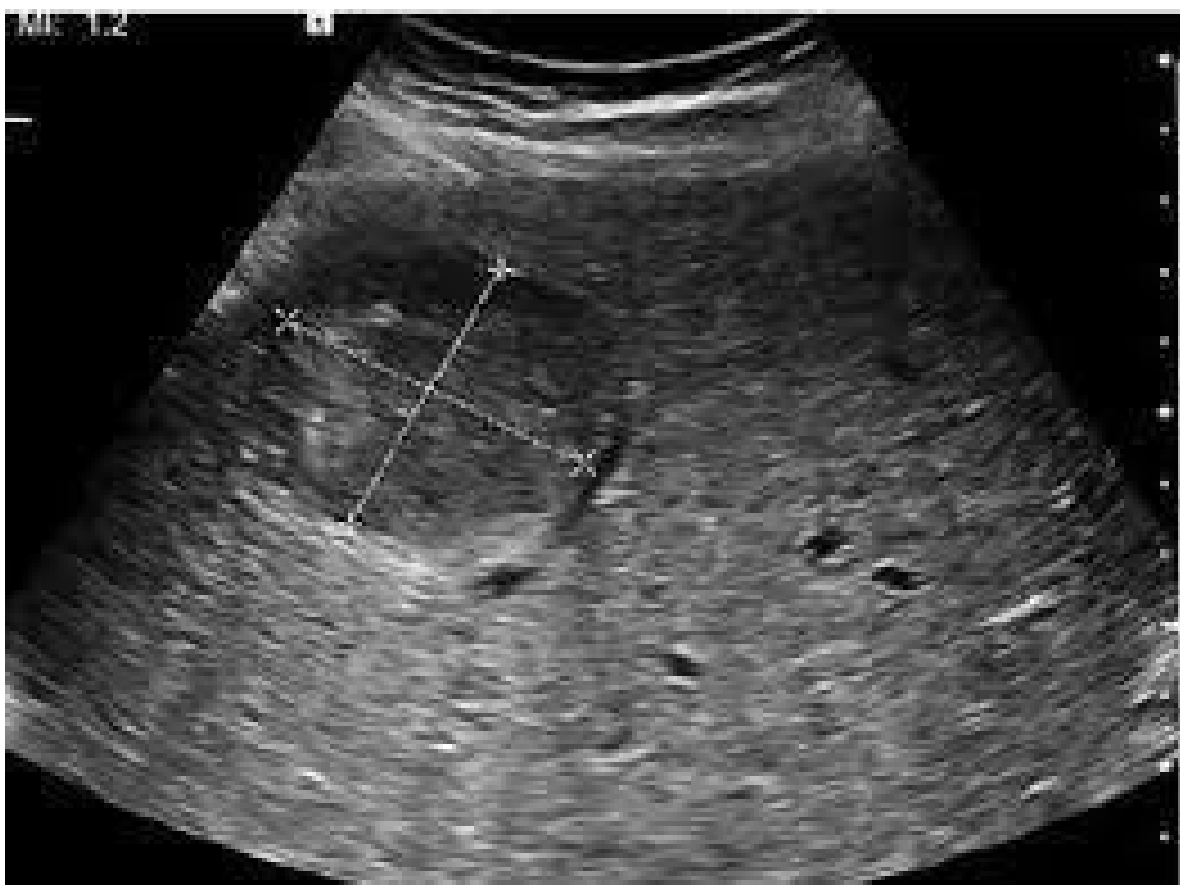

Figure 1.11 Ultrasound image of HCC (Sporea, 2014)

Computed tomography (CT) The research, differential diagnosis, phase-in and reaction assessment of hepatocellular carcinoma currently has an important role to play (HCC). HCC is the most frequently recorded CT-image hyper vascular tumour during the primarily arterial era of contrast improvement. Therefore, when examining this malignancy it is mandatory to use a spiral scanner to analyse the whole liver parenchyma correctly. The implementation of multi-phase protocols Contrast medium is essential for the imagery of focal liver lesions in CT after intravenous administration. Pictures are taken after 10 to 15 minutes at 40,60 and 180 seconds. (Hina ArifTiwari, 2014)

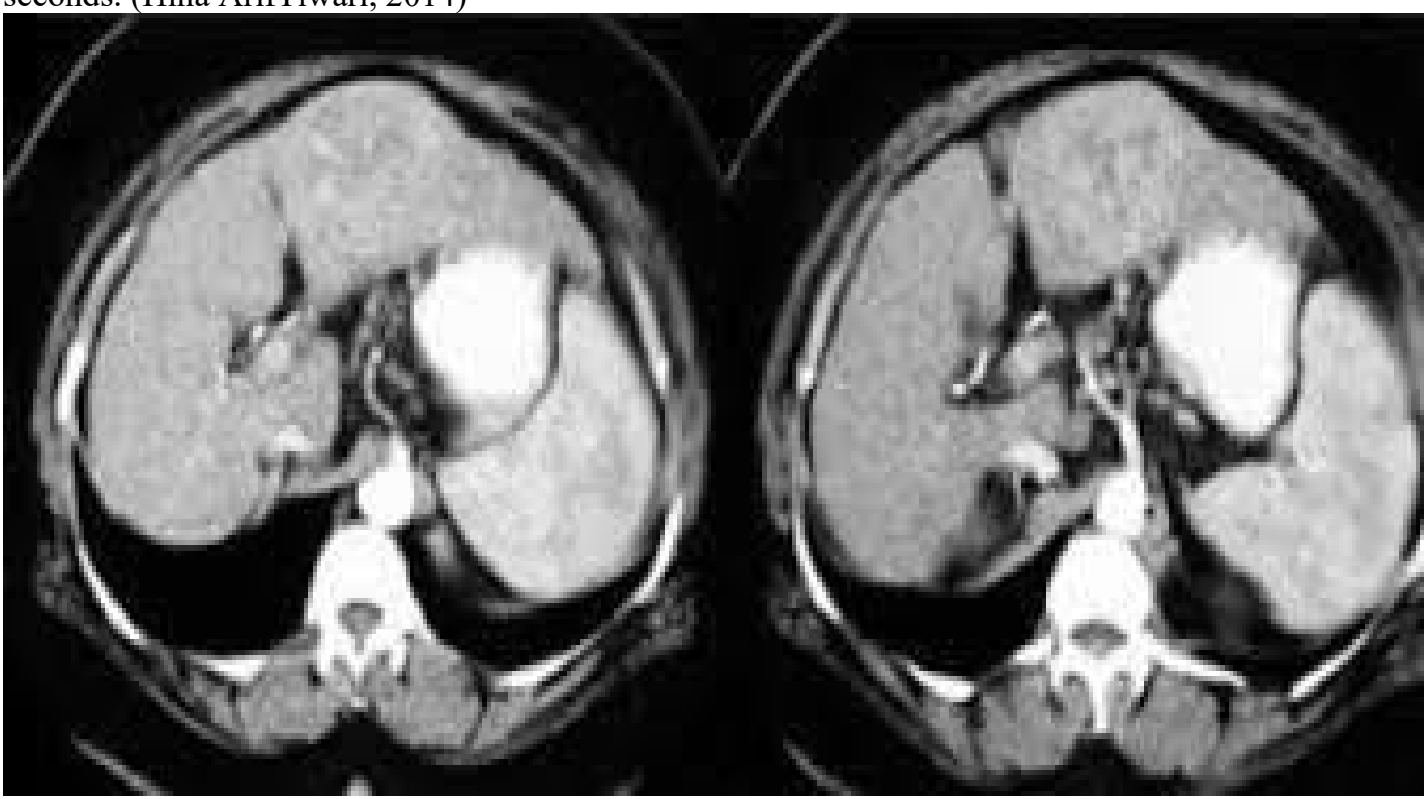

Figure1.12 CT scan image in the hepatic arterial phase of contrast enhancement showing neovascularity in a low-density hepatic mass (Jacobson, 2016)

Magnetic resonance imaging is a method of medical visualisation used in radiology to render representations of anatomy and internal functions of both health and illness of the body. MRI scanners produce physical images using high magnetic field, magnetic field gradient and radio waves. Most of them were MRI at 1.5 T. Higher fields like $3 \mathrm{~T}$ but now $7 \mathrm{~T}$ were more marketable because they were adaptive and had a greater resolution than before. Human experiments were conducted on up to 9,4 tonnes in labs, and animal studies were conducted on up to 21,1 tonnes. Several studies have shown that MRI is highly sensitive and precise

To detect HCC, especially in the case of tumours $1-2 \mathrm{~cm}$ tall. The benefits are ionising radiation protection, 
no chance of kidney injury, no reported cases of systemic nephrogenic fibrosis in patients with CFD, strong immunity to smaller tumours, increased tumour visualizations in the portal vein. On T1-weighted images, HCC appears with a variable signal from hyper intense to hypo intense. In the arterial process, irregularly growing HCC enhancement takes into account neoangiogenesis of the tumour and the supply of the blood from the hepatic artery. In the venous process of the portal, HCC is not smoother or starts to wash out and darker than the neighboring hepatic parenchyma. On pictures of $\mathrm{T} 2$

The context liver tends to be iso intense. 2 unique contrast media hepatic cyte are Gadolinium-based comparisons (Gadoexetate dimeglumine and gadobenate dimeglumine). Intravenous comparison bolus may be injected to provide information about hepatocytes after the injection at 20 or 60 years of age. The method used to measure blood flow, perfusion and vascularity of the tissue for dimensional comparison improved substance MRI is a technique used in MRI. The parameters achieved by DCE MRI are related to tumour micro-vessel density and endothelial vascular growth factor (VEGF). Focal HCC detection is based on complex multiphase 3D GRE, T1 and arterial step timing for HCC detection.
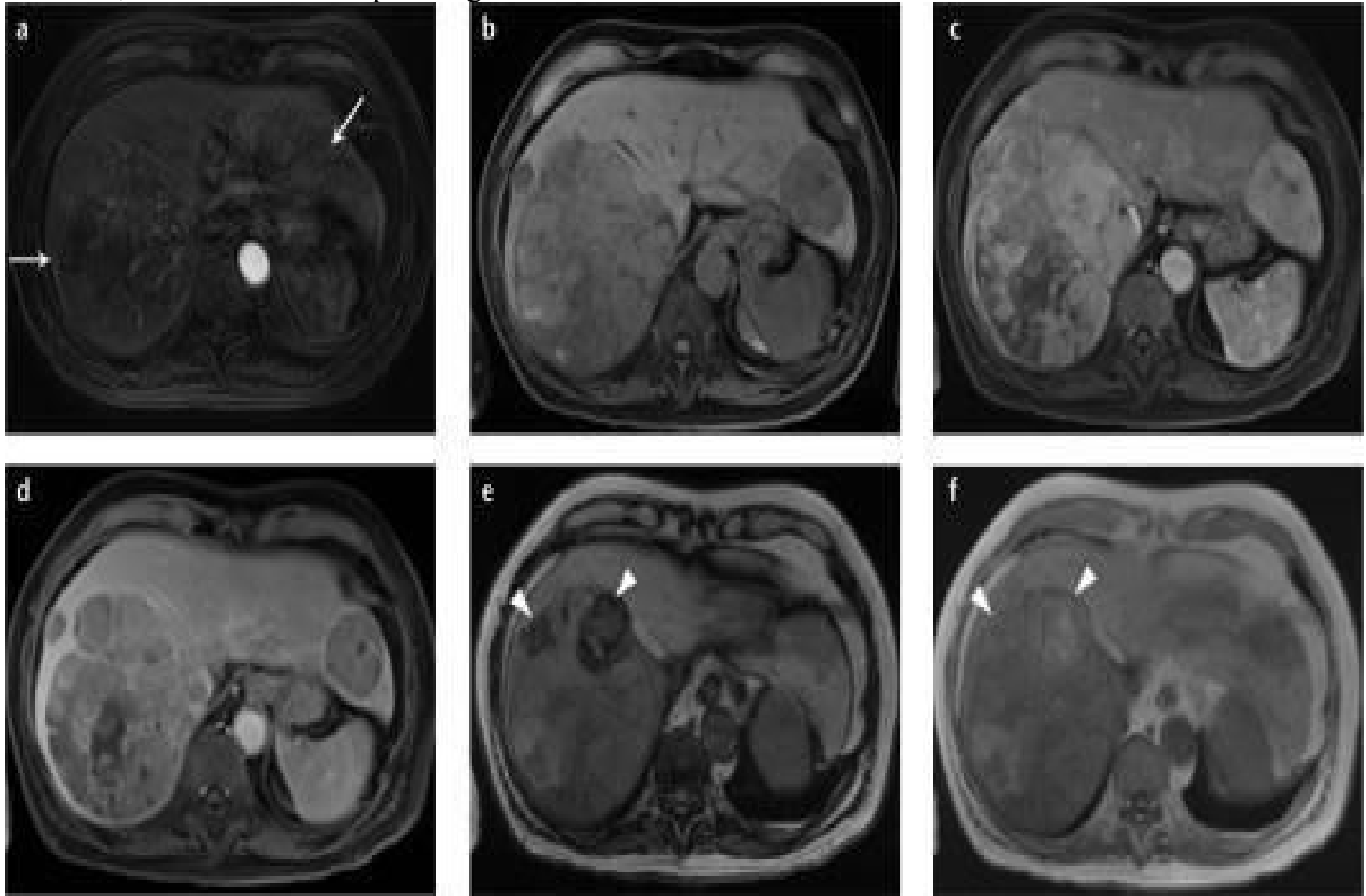

Figure1.14 (a) the normal vascularity of HCC cannot be shown because of the early contrast stage; MRI follow-up was then carried out with suitable timing. In the T1 weighted 3D GRE images, the pre contrast (b), arterial(c) and deferred phase(d) arterial enhancement in the two liver lobes is seen in multi tumors, following washout. In contrast to in-phases images (f, arrowheads), which consist of the internal fat within the tumour, the characteristic aspect of $\mathrm{HCC}$ is the absence of the signal in out-of-phase image (e, fletheads) (Hina Arif-Tiwari, 2014)

\section{MATERIAL AND METHOD}

The data was collected from the radiology department of AL Razi Health Care hospital, Lahore. It was a cross sectional and observational study. A sample of 96 patients had been selected using $5 \%$ level of significance and $10 \%$ margin of error as 0.5 anticipated proportion of frequency of hepatocellular carcinoma detection by gadolinium based contrast media on MRI. (Stephanie Channual, 2015). The study was conducted from August 2020 to November 2020. The Symptomatic patient presented with USG mass finding, Hepatitis c + , Cirrhosis, Splenomegaly, Right upper quadrant pain, Ascites, Yellow skin and eyes, Weight loss. The Pregnant females, children and clinically unstable patients who had received any contrast agent within 24 hours before and patients of cholangio carcinoma were excluded. SIEMENS

1.5 Tesla open bore Magnetic resonance imaging scanner was used. The patient was in supine position and the body coil was placed over the upper abdomen with cannulation of $18 \mathrm{G} / 20 \mathrm{G} / 22 \mathrm{G}$ and then three phases were obtained with contrast arterial, venous and hepatobiliary phase. The gadolinium based contrast media such as Magnevist, Omniscan or Gadovist was injected with the help of pressure. 


\section{STATISTICAL ANALYSIS}

Data were entered and analysed using version 23 of the Social Science Statistical Kit (SPSS). In the form of the median \pm SD and spectrum based on data distribution, all quantitative variables were provided.All the qualitative variables were presented in the form of frequency and percentages. Pie charts and bar charts were also used to display categorical data.

\section{RESULTS}

Ninety six (96) patients, who fulfilled the inclusion criteria, were included in the study. Out of which $62(64.6 \%)$ were males and 34(35.4\%) were females. (Table1)

\begin{tabular}{|lcc|}
\hline Gender & Frequency & Percent \\
\hline Male & 62 & 64.6 \\
\hline Female & 34 & 35.4 \\
\hline
\end{tabular}

\section{Table 1: Gender}

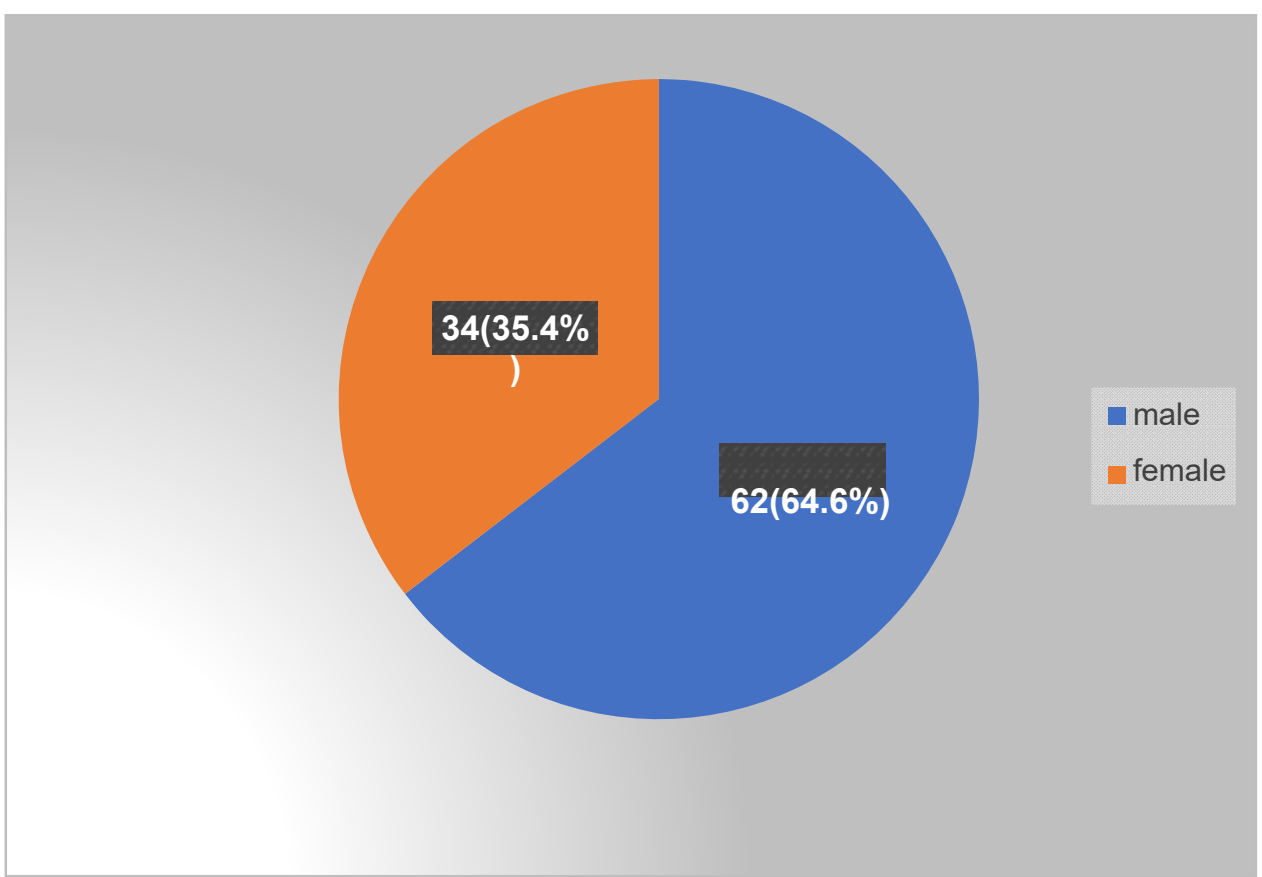

Figure 4.1 Genders of Patients

Patients were divided into 3 groups according to their age. Age groups and the frequency of patients in that group is given in table below. (Table 2)

\begin{tabular}{|lll|}
\hline Age & Frequency & Percent \\
\hline $\mathbf{1 0 - 3 0}$ & 15 & 15.6 \\
\hline $\mathbf{3 1 - 6 0}$ & 57 & 59.4 \\
\hline $\mathbf{6 1 - 8 0}$ & 24 & 25 \\
\hline
\end{tabular}

Table 2: Age distribution of patients 


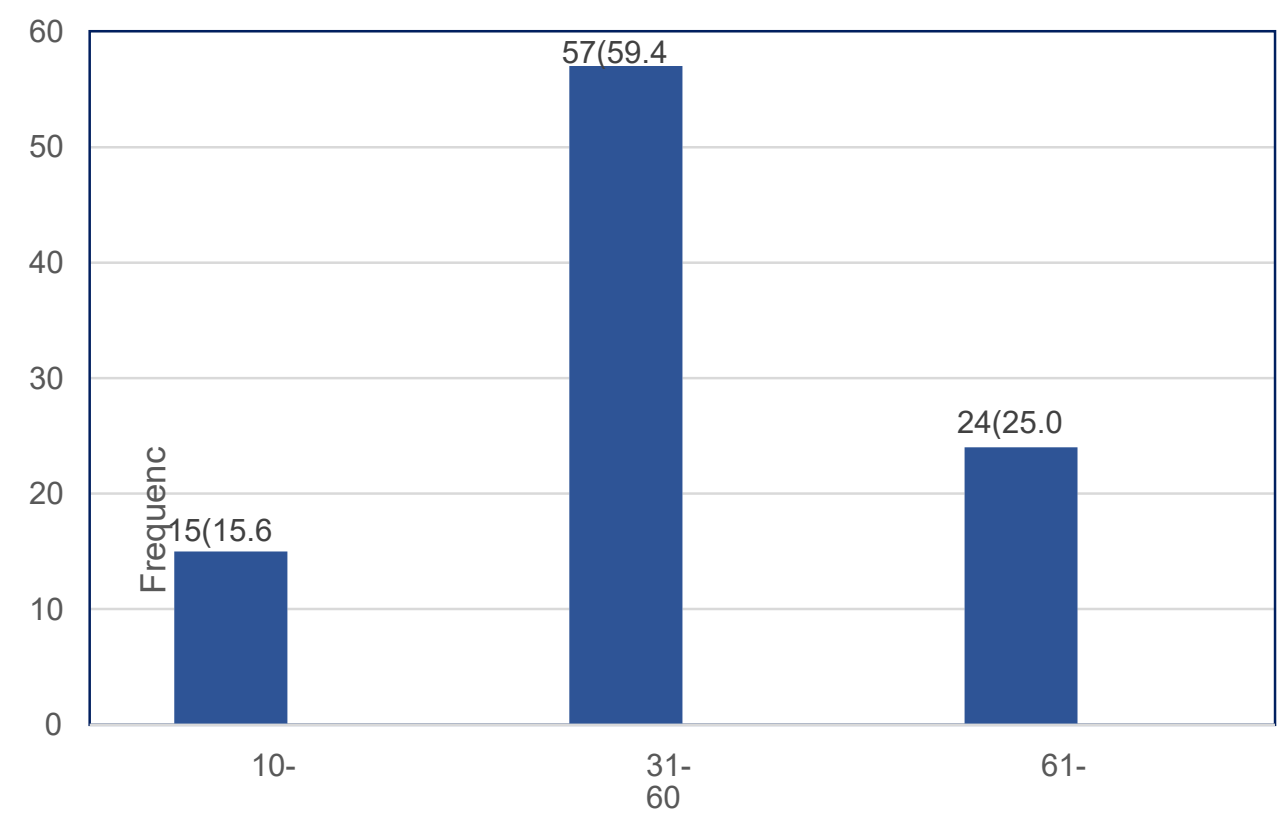

Figure 4.2 Age distribution of patients

Out of 96 patients 72 had USG finding mass. (Table 3)

$\begin{array}{cll}\text { USG finding mass } & \text { Frequency } & \text { Percent } \\ \text { Yes } & 72 & 75.0 \\ \text { No } & 24 & 25.0\end{array}$

Table 3: USG mass finding

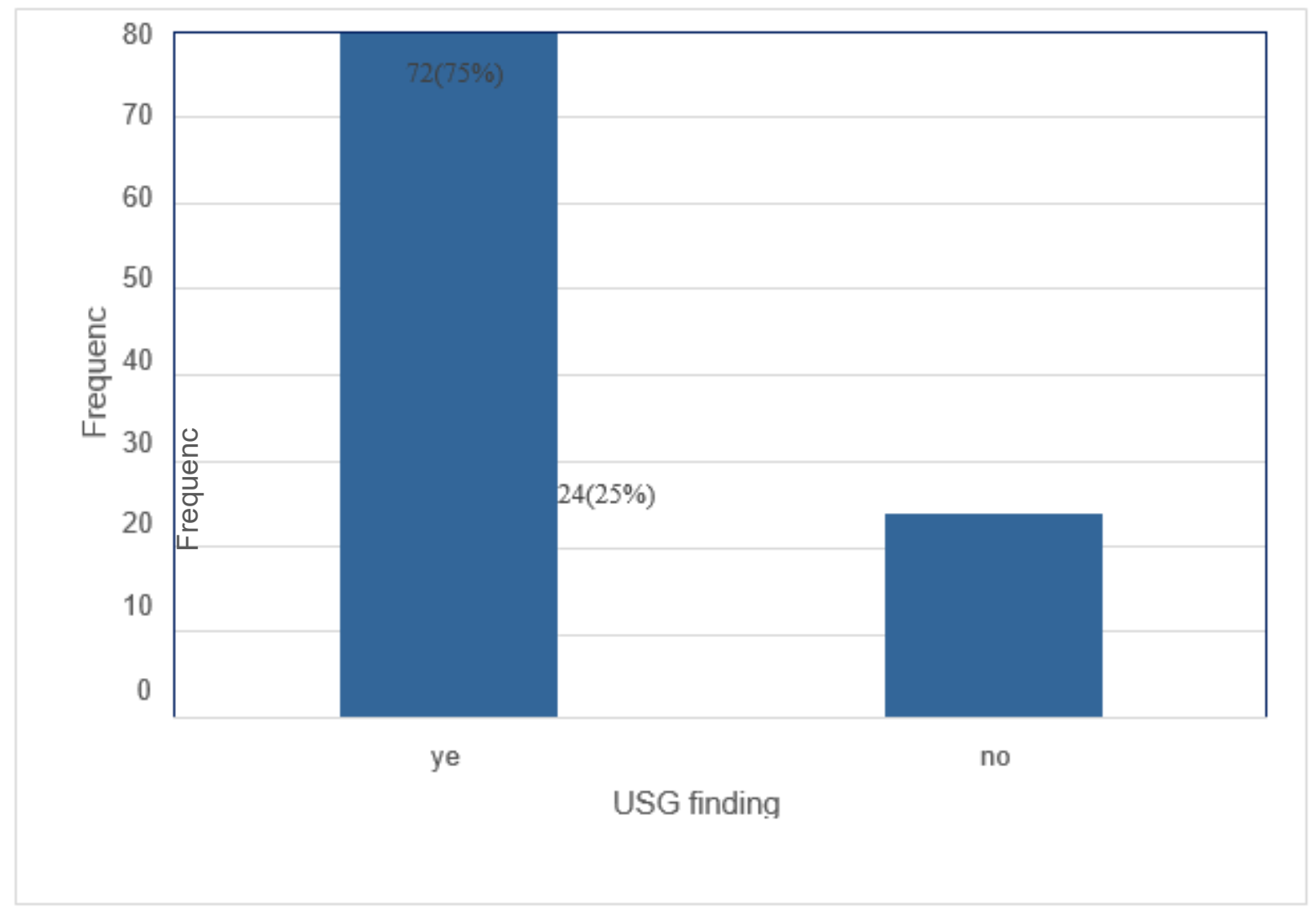

Figure 4.3 USG finding mass 
Out of 96 patients 62 were diagnosed with HCC. (Table 4)

\begin{tabular}{|lll|}
\hline MRI findings & Frequency & Percent \\
\hline Positive & 62 & 64.5 \\
\hline Negative & 34 & 35.4 \\
\hline
\end{tabular}

Table 4: MRI Findings

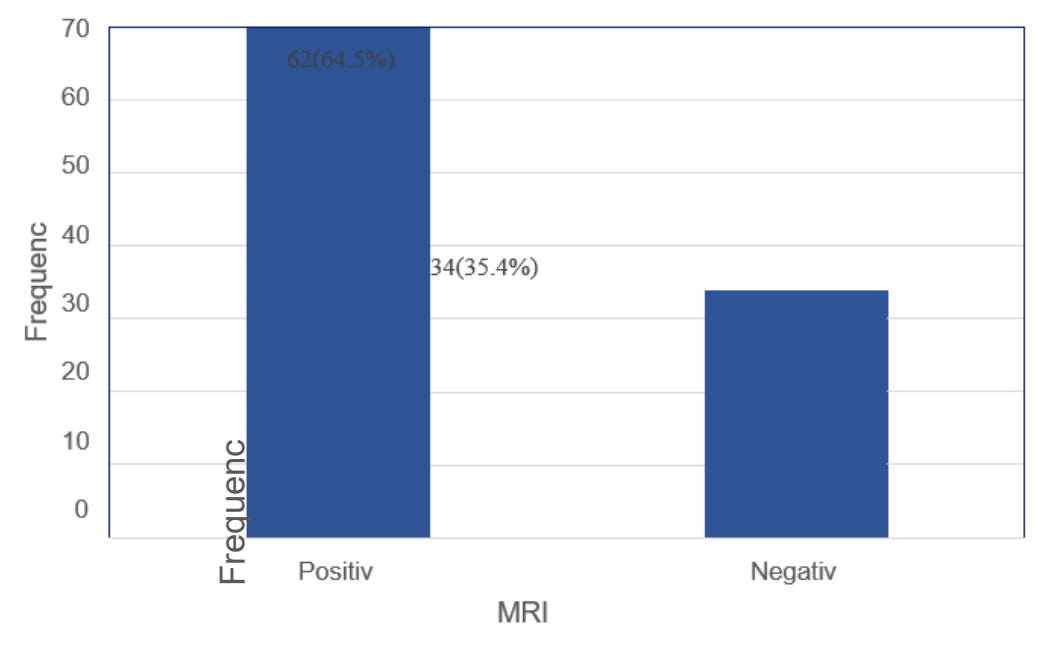

Figure 4.4 MRI findings

\section{DISCUSSION}

HCC is the world's most widespread malignancy. The higher frequency (mainly chronic hepatitis B and hepatitis C) of chronic viral hepatitis is due to the high prevalence in Asia.HCC differentiates itself from other vascular and cirrhotic lesions, including, inter alia, minor hemangiomas and hyper-vascular metastasis. In comparison with other imaging modalities, MRI offers a different category of details. By changing the pulse sequence parameters, tissues can be contrasted on the basis of the soothing properties of their hydrogen atoms.Multiple studies have provided the evidence for excellent sensitivity and specificity of MRI for the detection of HCC, especially for the tumors which are 1-2 cm in size. Gadolinium based contrast media (Gadoexetate dimeglumine and gadobenate dimeglumine) are 2 specific hepatocyte contrast media for liver study. Intravenous bolus of contrast are injected which will provide the information of hepatocytes at 20s or $60 \mathrm{~s}$ after the injection. Detection of focal HCC depends on dynamic, multiphase T1-weighted 3D GRE sequence and timing of arterial phase for detecting HCC. In this study ninety six (96) patients were taken as a sample size out of which 62 $(64.6 \%)$ were males and $34(35.4 \%)$ were females who fulfilled the criteria, with history of liver cirrhosis and USG mass finding were involved. The age of patients ranged from 10 to 80 years. Out of 96 patients $72(75 \%)$ had USG mass finding and $62(64.6 \%)$ were diagnosed with hepatocellular carcinoma as evaluated by gadolinium-based contrast media on MRI.

\section{CONCLUSION:}

In my study, $64.5 \%$ cirrhotic patients were found to have HCC as detected on MRI by using gadolinium-based contrast media. In past decades Biopsy was required for the conformation of HCC but now imaging evaluation especially MRI is consider enough for diagnosis of HCC. At appropriate time before disease progress into aggressive stage, early diagnosis will be beneficial, and more effective in treatment of HCC patients for better prognosis. HCC is an important cause of mortality in patients with chronic liver disease including patients with chronic hepatitis B. MRI is preferred over other modalities because it has advantages like safety from ionizing radiations, no risk of kidney damage, no known cases of nephrogenic systemic fibrosis in patients with Chronic Liver Disease, good sensitivity for smaller tumors with better visualization of tumor involvement of the portal vein. HCC appears with variable signal on T1-weighted images, ranging from hyper intense to hypo intense.

\section{REFERENCES}

1. Retrieved December 17, 2014, from Healthline: https://www.healthline.com/human- body-maps/right-lobeliver

2. Retrieved June 24, 2014, from Human Anatomy: http://msk- anatomy.blogspot.com/2013/06/lobes-ofliver.html

3. Retrieved August 22, 2017, from Simply Radiology: https://sugarytooth.wordpress.com/2017/08/22/liver/ 
4. Abul K. Abbas, J. C. (2013). Abul K. Abbas, Jon C Aster, MD PhD, VinayKumar

5. (9th ed.). Toronto: ELSEVIER.

6. Alzubaidi, S. J. (2018). Retrieved march 6, 2018, from Mayoclinic: https://www.mayoclinic.org/diseasesconditions/liver-cancer/doctors- departments/ddc-20353667

7. Am., S. C. (2014, May). Liver Anatomy. HHS public access, 4(90). Retrieved May 30, 2014, from NCBI:

8. https://www.ncbi.nlm.nih.gov/pmc/articles/PMC4038911/

9. assignmentpoint. (2018). Retrieved 208, from assignmentpoint: http://www.assignmentpoint.com/science/medical/hepatocellular- carcinoma.html

10. Daley, B. (2016). Retrieved September 12, 2016, from Radiology Affiliates Imaging: https://4rai.com/blog/why-the-3-tesla-mri-is-the-best-scanner-for-diagnostic-imaging

11. Damzyxno. (2018). LIVER: Anatomy, Physiology, Pathology and Care. Retrieved january 2018, from steemit beta: https://steemit.com/steemstem/@damzxyno/liver- anatomy-physiology- pathology-and-care

12. Hina Arif-Tiwari, B. K. (2014, April). https://www.ncbi.nlm.nih.gov/pmc/articles/PMC4463351/. Diagnostic and interventional radiology, 20(3).

13. Hoffman, K. (2016). Retrieved December 17, 2016, from Medivizor: https://medivizor.com/blog/2016/12/17/liver/

14. Ichikawa T, S. K. (2010, march). Detection and characterization of focal liver lesion.

15. Wolters Kluwer, 45(3).

16. Jacobson, D. R. (2016). Retrieved April 8, 2016, from Medscape: https://emedicine.medscape.com/article/369226-overview

17. Julius balogh, D. V.(2016, October). Hepatocellular carcinoma . J Hepatocellualr carcinoma, 3(41-53).

18. Keith L. Moore, A. F. (2014). Clinically oriented Anatomy (7th ed.). Toronto: Wolters Kluwer.

19. MasatakeTanaka, Y. (2016, November). The Hepatic Lymphatic Vascular System: Structure, Function, Markers, and Lymphangiogenesis. ELSEVIER, 2(6).

20. May, H. (2018). Retrieved August 7, 2018, from Teachmeanatomy: https://teachmeanatomy.info/abdomen/viscera/liver/

21. Michael Reid, J. C. (2017, December). HCV in the Older Patient - Natural history, extrahepatic disorders, safety and efficacy of treatment regimens. 31(4).

22. Park MJ, Y. K. (2013, march). Validation of diagnostic criteria using gadoxetic acid- enhanced and diffusion weighted MR imaging for small hepatocellular carcinoma $(<=2.0 \mathrm{~cm})$ in patients with hepatitis induced liver cirrhosis . SAGE journal, 10.

23. Runyon, C. (2016). Retrieved april 5, 2016, from amberusa: https://www.amberusa.com/blog/howultrasound-machines-work

24. Sporea, L. (2014). Retrieved June 2014, from ResearchGate: https://www.researchgate.net/figure/CEUSexamination-in-a-patient-with- hepatocellular-carcinoma-HCC-a-HCC-aspect-in_fig3_262050680

25. Stephanie Channual, N. T. (2015, january ). Gadoxetate Dissodium-enhanced MRI to Differentiate Dysplastic nodules and grade of hepatocellular carcinoma: correlation with histopathology. American journal roentgenology, 205.

26. T. Vescovo, G. R. (2016, October). Molecular mechanisms of hepatitis C virus- induced hepatocellular carcinoma. Science Direct, 22(10).

27. Wu LM, X. J. (2013). Is liver-specific gadoxetic acid enhanced magnetic resonance imaging a reliable tool for detection of hepatocellular carcinoma in patients with chronic liver disease? Digestive disease and science, 58(11).

28. Young Kon Kim, C. S. (2003, August). Comparison of Superparamagnetic Iron Oxide-Enhanced andGadobenate Dimeglumine-Enhanced Dynamic MRI forDetection of Small Hepatocellular carcinoma. American journalroentgenology, 18 